\title{
Long non-coding RNA XIST promotes cerebral ischemia/reperfusion injury by modulating miR-27a-3p/FOXO3 signaling
}

\author{
HUA ZHANG, JUNYONG XIA, QIUSHAN HU, LIQIN XU, HONGYAN CAO, XU WANG and MIN CAO \\ Department of Neurosurgery, China Resources \& WISCO General Hospital (CR \& WISCO General Hospital \\ Affiliated to Wuhan University of Science and Technology), Wuhan, Hubei 430080, P.R. China
}

Received September 22, 2020; Accepted March 30, 2021

DOI: $10.3892 / \mathrm{mmr} .2021 .12205$

\begin{abstract}
Cerebral ischemia/reperfusion (I/R) injury leads to neuronal damage, which may cause disability and even mortality. Multiple studies have revealed that long non-coding RNAs (lncRNAs) serve pivotal roles in the pathogenesis of cerebral I/R injury. Therefore, the present study aimed to investigate whether the lncRNA $\mathrm{X}$ inactivate-specific transcript (XIST) protects neuronal cells from cerebral I/R injury. In the present study, reverse transcription-quantitative PCR demonstrated that XIST expression was upregulated in the brain tissues of an I/R mouse model and in oxygen and glucose deprivation/reperfusion (OGD/R)-treated Neuro-2a (N2a) cells. Knockdown of XIST alleviated cerebral injury, as well as reduced $\mathrm{N} 2 \mathrm{a}$ cell apoptosis and reactive oxygen species (ROS) production. Additionally, luciferase reporter and RNA immunoprecipitation assays identified that XIST could bind with microRNA (miR)-27a-3p. It was found that miR-27a-3p expression was downregulated in the brain tissues of an $\mathrm{I} / \mathrm{R}$ mouse model and in OGD/R-induced N2a cells. In addition, miR-27a-3p overexpression attenuated I/R-induced cerebral injury, and inhibited the apoptosis and ROS production of N2a cells. miR-27a-3p was found to target FOXO3. Silencing of FOXO3 alleviated cerebral injury, as well as inhibited N2a cell apoptosis and ROS production. Collectively, these findings indicated that XIST aggravated cerebral I/R injury by regulating miR-27a-3p/FOXO3 signaling, which may provide a novel insight into the treatment of cerebral I/R injury.
\end{abstract}

Correspondence to: Dr Xu Wang, Department of Neurosurgery, China Resources \& WISCO General Hospital (CR \& WISCO General Hospital Affiliated to Wuhan University of Science and Technology), 29 Yejin Road, Qingshan, Wuhan, Hubei 430080, P.R. China

E-mail: wangxu8696@hotmail.com

Key words: $\mathrm{X}$ inactivate-specific transcript, microRNA-27a-3p, FOXO3, cerebral ischemia/reperfusion injury

\section{Introduction}

Ischemic stroke is a major risk factor for human health and causes cerebral injury, disability and even mortality worldwide (1). It is estimated that ischemic stroke accounts for $70 \%$ of all types of strokes $(2,3)$. Ischemia reperfusion (I/R) mainly contributes to the repair and functional recovery of damaged tissues and organs. However, occasionally, I/R aggravates the dysfunction of tissues and organs and promotes structural damage, which is known as $\mathrm{I} / \mathrm{R}$ injury $(4,5)$. Cerebral I/R leads to mitochondrial dysfunction, inflammation, excessive release of reactive oxygen species (ROS), massive glutamate excitotoxicity and cell death, and finally irreversible damage to the brain (6). The inflammatory response and apoptosis are the major causes of neuronal cell injury after cerebral I/R (7). Currently, the clinical therapeutic strategies for ischemic stroke remain unsatisfactory. Thus, it is necessary to investigate the molecular mechanisms underlying the inflammatory response and neuronal apoptosis for the treatment of I/R injury.

Long non-coding RNAs (lncRNAs), which are $>200$ nucleotides in length, lack complete open reading frames and are incapable of coding proteins $(8,9)$. An increasing number of studies have revealed that lncRNAs are involved in various physiological and pathological processes of diseases (10-12). Previous studies have verified that the lncRNA $X$ inactivate-specific transcript (XIST) participates in the progression of several diseases. For instance, XIST promoted cisplatin resistance in human lung adenocarcinoma cells by modulating the let-7i/BAG cochaperone 1 axis (13). XIST also facilitated the proliferation of human fibroblasts and murine lung fibroblasts, as well as extracellular matrix protein expression by sponging microRNA (miRNA/miR)-139 (14). Moreover, XIST triggered neuropathic pain by binding with miR-544 to activate STAT3 in a rat model of chronic constriction injury (15). Nevertheless, the molecular roles and the regulatory mechanisms of XIST in cerebral I/R injury have not been fully elucidated.

The present study aimed to investigate the molecular mechanism of XIST in cerebral I/R injury by establishing an I/R mouse model in vivo and an oxygen and glucose deprivation/reperfusion (OGD/R)-treated Neuro-2a (N2a) cellular model in vitro. The findings of the present study may shed new light on the treatment of cerebral I/R injury. 


\section{Materials and methods}

Experimental animals and middle cerebral artery occlusion (MCAO) model. All animal experiments were performed with the approval and in accordance with standard principles approved by the Institutional Animal Care and Use Committee of China Resources \& WISCO General Hospital (approval no. 2020-005; Hubei, China). The Experimental Animal Center of the Chinese Academy of Medical Sciences (Beijing, China) provided 90 male C57BL/6J mice (age, 8-10 weeks; body weight, 22-30 g) for the current experiments. The mice were maintained at $25^{\circ} \mathrm{C}$ in $40-60 \%$ humidity on a $12 \mathrm{~h}$ light/dark cycle with free access to food and water. All mice were divided into seven groups: i) Sham + Adeno-associated virus (AAV)-short hairpin RNA (sh)-negative control (NC) group (n=20); ii) I/R+ AAV-sh-NC group ( $n=20)$; iii) I/R + AAV-sh-XIST group (n=10); iv) I/R + AAV-sh-FOXO3 group $(\mathrm{n}=10)$; v) sham + AAV-NC mimic group $(\mathrm{n}=10)$; vi) $\mathrm{I} / \mathrm{R}+$ AAV-NC mimic group ( $n=10)$; and vii) $\mathrm{I} / \mathrm{R}+\mathrm{AAV}-\mathrm{miR}-27 \mathrm{a}-3 \mathrm{p}$ mimic group $(n=10)$.

In all $\mathrm{I} / \mathrm{R}$ groups, mice were subjected to MCAO. After anesthesia with pentobarbital sodium $(45 \mathrm{mg} / \mathrm{kg})$, a monofilament nylon suture was inserted into the internal carotid artery through the external carotid artery, followed by occluding the origin of the MCA. The occlusion continued for $2 \mathrm{~h}$, and the suture was slowly removed to allow blood flow and induce reperfusion. The mice in the sham group were treated in the same way, but without occlusion of the MCA. Mice that died or failed to show an $80 \%$ reduction in cerebral blood flow after occlusion were excluded from the following experiments. A total of $24 \mathrm{~h}$ after reperfusion, measurements, including Longa's scores, cerebral blood flow and infarct volume, were conducted. The experiments were performed for $\sim 26 \mathrm{~h}$, with $2 \mathrm{~h}$ for occlusion and $24 \mathrm{~h}$ for reperfusion. The health and behavior of rats were monitored every $6 \mathrm{~h}$ during reperfusion. Animals $(n=90)$ were immediately euthanized when they showed signs of weight loss $>20 \%$, dehydration or were non-ambulate, using intraperitoneal injection of $45 \mathrm{mg} / \mathrm{kg}$ pentobarbital sodium followed by cervical dislocation. Mouse death $(n=90)$ was confirmed by bilateral thoracotomy and the subsequent absence of pupillary response, respiratory movement and heartbeat. When the I/R model was successfully established, the brain tissues were instantly collected and used for detection of the RNA expression.

$A A V$ delivery. AAVs (serotype 9; at a titer of $3.5 \times 10^{12} \mathrm{vg} / \mathrm{ml}$ ) carrying short hairpin RNA (sh)-negative control (NC), shXIST, sh-FOXO3, NC mimic and miR-27a-3p mimic were constructed by Shanghai GeneChem Co., Ltd. AAV-sh-NC served as the control for AAV-sh-XIST and AAV-sh-FOXO3, and the AAV-NC mimic served as the control for the AAV-miR-27a-3p mimic. A total of 14 days before establishment of the MCAO model, $3.5 \times 10^{10} \mathrm{v} . \mathrm{g} . /$ mouse were stereotactically injected into the ipsilateral lateral ventricle (coordinated from bregma) of the mice using a 10- $\mu$ l Hamilton syringe and the needle was maintained for $10 \mathrm{~min}$ before retraction.

Evaluation of Longa's scores. After cerebral I/R injury, the neurological deficit scores of the mice were evaluated.
Mice that underwent MCAO were allowed to rest for $24 \mathrm{~h}$ before the evaluation. Neurological scores were assessed at 24, 48 and $72 \mathrm{~h}$ after MCAO by technicians blinded to the treatment groups. Longa neurological examination was divided into five grades as previously described (16): i) 0 , normal, without neurological deficits; ii) 1 , the limb could not completely extend to the left, with mild neurological deficits; iii) 2 , movement made in a 0 , with moderate neurological deficits; iv) 3, left descent, with severe neurological deficits; v) 4, unable to walk and loss of consciousness; and vi) 5, death.

Measurement of infarct volume. The brain tissue was sectioned, fixed with $4 \%$ paraformaldehyde at room temperature for $24 \mathrm{~h}$, embedded in paraffin and then cut into coronal sections. Infarct volume was measured on five slices of 3-mm coronal sections from each brain. A 2\% 2,3,5-triphenyltetrazolium chloride (TTC; cat. no. T8877; Sigma-Aldrich; Merck $\mathrm{KGaA}$ ) solution was incubated with the slices for $20 \mathrm{~min}$ at $37^{\circ} \mathrm{C}$. Stained images were captured with a camera, and the infarct area was measured with ImageJ version 6.0 software (National Institutes of Health). For minimization of the effect of brain edema, the infarct volume was calculated with the following formula: $100 \% \mathrm{x}$ (contralateral hemisphere volume-non-infarct ipsilateral hemisphere volume)/contralateral hemisphere volume.

Measurement of cerebral blood flow. For the measurement of the cerebral blood flow of mice, a laser Doppler flowmetry was used as previously described (17). Briefly, the head of the mouse was fixed using a stereotaxic device. Then, an incision was made in the skin to cover the calvarium to expose the bregma. Finally, the laser Doppler flow probe was placed to dynamically measure cerebral blood flow for $2 \mathrm{~h}$. Cerebral blood flow decreasing to $\geq 20 \%$ of baseline during occlusion indicated successful establishment of the cerebral I/R injury mouse model.

Bioinformatics analysis. miRNAs with potential binding sites for XIST were predicted using the online software program DIANA (lncBase v.2; http://carolina.imis.athena-innovation. gr/diana_tools/web/index.php?r=lncbasev2\%2Findex-predi cted). miR-27a-3p was predicted to possess a binding site with XIST. The target genes of miR-27a-3p were identified using the TargetScan database (version 7.2; http://www.targetscan.org/). miR-27a-3p was revealed to bind with the 3'UTR of FOXO3.

$N 2$ a cell culture and $O G D / R$ treatment. OGD/R-treated N2a cell models can be used as in vitro models of cerebral ischemia/reperfusion (18-20). The mouse neuroblastoma cell line N2a was obtained from the American Type Culture Collection, and was cultured in DMEM (Gibco; Thermo Fisher Scientific, Inc.) containing 10\% FBS (Gibco; Thermo Fisher Scientific, Inc.), $1 \%$ penicillin/streptomycin (Gibco; Thermo Fisher Scientific, Inc.) at $37^{\circ} \mathrm{C}$ with $5 \% \mathrm{CO}_{2}$.

To mimic in vitro I/R injury conditions, N2a cells were transferred to glucose-free DMEM and incubated for $2 \mathrm{~h}$ in a hypoxic atmosphere containing $5 \% \mathrm{CO}_{2}, 1 \% \mathrm{O}_{2}$ and $94 \% \mathrm{~N}_{2}$. Finally, N2a cells were collected after reoxygenation in normal culture medium and maintained at $37^{\circ} \mathrm{C}$ for $24 \mathrm{~h}$ under normoxic conditions $\left(5 \% \mathrm{CO}_{2}\right)$ for further experiments. 
Cell transfection. shRNAs against XIST and FOXO3, as well as the corresponding sh-NC, were synthesized by Sangon Biotech Co., Ltd. miR-27a-3p mimics and inhibitors, as well as their corresponding NCs (NC mimics, NC inhibitor; at a concentration of $5 \mu \mathrm{M}$ ), pcDNA3.1/FOXO3, pcDNA3.1 and pcDNA3.1/XIST were purchased from Shanghai GenePharma Co., Ltd. Lipofectamine ${ }^{\circledR} 2000$ (Invitrogen; Thermo Fisher Scientific, Inc.) was used for transfection of the aforementioned oligonucleotides $(40 \mathrm{mM})$ or plasmids $(2 \mu \mathrm{g})$ into $\mathrm{N} 2 \mathrm{a}$ cells at room temperature for $6 \mathrm{~h}$ and cells were then incubated for another $24 \mathrm{~h}$ before subsequent experiments. The sequences of sh-XIST, sh-NC, sh-FOXO3, miR-27a-3p mimics and inhibitor, NC mimics, NC inhibitor, pcDNA3.1/FOXO3, pcDNA3.1 and pcDNA3.1/XIST are provided in Tables I-III. The cells were harvested after $48 \mathrm{~h}$, and reverse transcription-quantitative (RT-q) PCR was used to measure the expression levels of XIST, FOXO3 and miR-27a-3p. GAPDH and U6 served as the internal controls.

$R N A$ extraction and $R T-q P C R$. TRIzol ${ }^{\circledR}$ reagent (Invitrogen; Thermo Fisher Scientific, Inc.) was used to extract total RNA from brain tissues and N2a cells. A NanoDrop ${ }^{\mathrm{TM}} 2000$ spectrophotometer (Thermo Fisher Scientific, Inc.) was used to detect the RNA concentrations. Then, $1 \mu \mathrm{g}$ RNA was reverse transcribed into cDNA using a PrimeScript ${ }^{\mathrm{TM}}$ RT kit (cat. no. RR037A; Takara Bio, Inc.) for lncRNA and mRNA, or the TaqMan ${ }^{\mathrm{TM}}$ miRNA RT kit (cat. no. 4366596; Applied Biosystems; Thermo Fisher Scientific, Inc.) for miRNA, according to the manufacturer's instructions. RT-qPCR was conducted to amplify XIST, miR-27a-3p and FOXO3 using a SYBR-Green Real-Time PCR kit (Takara Bio, Inc.) under the following thermocycling conditions: Initial denaturation at $95^{\circ} \mathrm{C}$ for $30 \mathrm{sec}$, followed by 35 cycles of denaturation at $95^{\circ} \mathrm{C}$ for $5 \mathrm{sec}$, annealing at $55^{\circ} \mathrm{C}$ for $20 \mathrm{sec}$ and at $72^{\circ} \mathrm{C}$ for $20 \mathrm{sec}$, and final extension at $72^{\circ} \mathrm{C}$ for $3 \mathrm{~min}$. The relative expression levels of XIST, miR-27a-3p and FOXO3 were calculated using the $2^{-\Delta \Delta \mathrm{Cq}}$ method (21) with GAPDH as an internal control for XIST and FOXO3, and U6 as an endogenous control for miR-27a-3p. The primer sequences were as follows: XIST forward (F), 5'-GGTTCTGTCAAGATACTTTCCT-3' and reverse (R), 5'-CAATGAAGAGCTTGACGTG-3'; miR-27a-3p F, 5'-TTCACAGTGGCTAAGTTCCGC-3' and R, 5'-CTCTAC AGCTATATTGCCAGCCAC-3'; FOXO3 F, 5'-ATCTACGAG TGGATGGTGC-3' and R, 5'-CCGGATGGAGTTCTTCCA G-3'; GAPDH F, 5'-GATCATCAGCAATGCCTCC-3' and R, 5'-TCCACGATACCAAAGTTGTC-3'; and U6 F, 5'-CAATAC AGAGAAAGTTAGCACG-3' and R, 5'-AATGCTTCAAAG AGTTGTGC-3'.

Flow cytometry. N2a cell apoptosis was detected via flow cytometry using an Annexin V/PI double staining kit (BD Biosciences) according to the manufacturer's instructions. N2a cells were collected and then washed twice with ice-cold PBS. Next, the cells were resuspended in binding buffer and stained with Annexin V-FITC and PI at room temperature for 15 min in the dark. Finally, flow cytometry was performed with a Coulter ${ }^{\circledR}$ EPICS XL instrument (Beckman Coulter, Inc.). The data of flow cytometry were analyzed using Flowing version 2.5.1 software (Turku Bioscience) and Origin version 8 software (OriginLab). The apoptosis rate was calculated as the percentage of early apoptotic cells to total cells.
Table I. Relative sequences of shRNAs.

\begin{tabular}{lc}
\hline shRNAs & Sequences $\left(5^{\prime} \rightarrow^{\prime}\right)$ \\
\hline sh-XIST & ACCTTCTGCGTGTTCATTATT \\
sh-NC & ATCTTAATGTTTTCGGCTCCT \\
sh-FOXO3 & TTTCATCGAGTTCCCGACTCG \\
sh-NC & GTCTTAATCCGTGGTTCCCCA
\end{tabular}

shRNA/sh, short hairpin RNA; NC, negative control; XIST, X inactivate-specific transcript.

Table II. Relative sequences of miRNA mimics and inhibitor.

miRNA mimics/

inhibitor Sequences $\left(5^{\prime} \rightarrow 3^{\prime}\right)$

miR-27a-3p mimics CCACCAUCGGUUUAGCGUAUG

NC mimics GUGAGGACCACCUGUCUCAUU miR-27a-3p inhibitor GGUUUACCUUCCCGGGACAAU $\mathrm{NC}$ inhibitor AUCGGCCUACGUGUAUUCGCA

miRNA/miR, microRNA; NC, negative control.

Western blot analysis. Western blot analysis was repeated three times in this study. Total proteins from brain tissues and N2a cells were extracted using RIPA lysis buffer (Beyotime Institute of Biotechnology) with protease inhibitor [Roche Diagnostics (Shanghai) Co., Ltd.]. The protein concentration was determined using a BCA kit (Beyotime Institute of Biotechnology). Then, the proteins (30 $\mu \mathrm{g}$ per lane) were separated by $10 \%$ SDS-PAGE and further transferred onto PVDF membranes. After the membranes were blocked with $5 \%$ skimmed milk for $2 \mathrm{~h}$ at room temperature, they were cultured with anti-FOXO3 (cat. no. ab70315; 1:2,000; Abcam) and anti-GAPDH (cat. no. ab9485; 1:2,500; Abcam) antibodies overnight at $4^{\circ} \mathrm{C}$. Then, the membrane was incubated with a goat anti-rabbit HRP-conjugated secondary antibody (cat. no. ab205718; 1:2,000; Abcam) at room temperature for $1 \mathrm{~h}$. The signals were visualized using a chemiluminescence imaging system and chemiluminescence detection reagents (Bio-Rad Laboratories, Inc.) with GAPDH as the internal control. The Quantity One software (4.5.0 basic; Bio-Rad Laboratories, Inc.) was used for the semi-quantification of protein expression.

RNA immunoprecipitation (RIP) assay. The RIP assay was conducted using a Magna RNA-binding protein immunoprecipitation kit (EMD Millipore) following the manufacturer's instructions. N2a cells $\left(2 \times 10^{7}\right)$ after indicated transfection were cross-linked with $0.75 \%$ formaldehyde. Chromatin was then sheared by sonication at $16,000 \mathrm{x}$ g for $10 \mathrm{~min}$ at $4^{\circ} \mathrm{C}$. N2a cells were lysed with RIPA lysis buffer (cat. no. R0278; Sigma-Aldrich; Merck KGaA) for $5 \mathrm{~min}$ on ice and then centrifuged at $16,000 \times \mathrm{g}$ at $4^{\circ} \mathrm{C}$ for $10 \mathrm{~min}$. Briefly, $\mathrm{N} 2 \mathrm{a}$ cells anti-argonaute2 (Ago2; cat. no. 2897S; 1:1,000; Cell Signaling Technology, Inc.) and anti-IgG (cat. no. 14678-1-AP; 
Table III. Relative sequences of pcDNA3.1/RNAs.

pcDNA3.1/RNAs

Sequences $\left(5^{\prime} \rightarrow 3^{\prime}\right)$

pcDNA3.1/XIST gagttggetgttttcccegccgeccetgcacettcgtttaactttagtgatttcttcegtcgtacactttcattttagatcgttaccetcceggtgtgacttcctt gcattatccgtgattttggggtcttactattctcttcttttgcgatttaggagctttctcgccatattttgaccgatcttctatattccgcetttgtttaactcatttttg tatctggtctttttttatgtcetcegctcatctggettctatgggtcgttcttggegegtctctcttgatttcettgtctttgttgtgacgetggaccatcttcgtctt tactggtgcaagttagattgttttctttcttctacgagtctcetcetttctctgcaagtttttttcttatttttcggtctctggttacttacgtettgttttcttgtgttta cttttcgttgetttgatttttgegttcccattcetttgtaccttattggtgttgtagggettttctatttggttggttattttcetcttatagatttcgectggttatttgtc ttctttttatattcttgattatctttttttcttctgtttcttccggaggttgttcttttcetctctgtatgtcgecttgtcattttttttcgagcattttgctctccagttgtc ccagtgttttcacctcttttttttgtagccggttatattgctatttatcagacgtattcgtcttttttgttggaattttctattcgtccgttgtttgatttttgcttgtgata attttttttttacttttgcctgctttcttcgtgtcctcttgttcctagtattctgagttttcatctggtggtccgtccgettgcctttacctcttgtcetcc

pcDNA3.1 gtggtagggectagctcgagggaacctaccagctgttgegcctcgctgacagcgtcgtcetgtctattcgagcegcgggacagctctccegcgtggt gttcattccegtgtgactagtgggccgtgtaccgcctaacgecggtggcagcgcacgttccagaccgcagcggctttcacgaggcaaccgcatgtgt agactaaacagcttcagtatcagcccegggtccactttcggtgattgtcagacgegtgctctctcgaagcaagaacttaggagggctcagggggecgc tccattgtgcaacgactatcgcgttgtcettcgtcgetggegccgtggtgaatacacatccgacccgctatctgtgtaggtaaatcaccgggtgggtgcc tccggcggcgaccegtaggtcgcactctaacttattagtaggaactcgtgaagggtgtccacgttatgaaacttagtcccctacagcgegcgtctagcg accccgcgatggggacattgagagctggctcgagatattcgcccgccaagggtccggagaaggettggtgctggagtggagaagtgaaccgaaga tttcgggggtccgaagccgtgtggtatcgaagtacctagttagtctcgtgcgtttccgttgatgagcaagcaagccccatcgggacccgatcgtaacac agtgttgccgtgacgttgggagccetgattagccggtttactgccatcgcagggtcgtgctaggtgggctccagctgcacgggtcgtcgctatctgccc tgccggtcg

pcDNA3.1/FOXO3 agtttcattactatcgcgacaacctaacgatttgcattcatctggggttggacactgccceggcctaaagaactgaccacgactacacatggtagattcgc cetccacctcattctcgtacgacacacacattcaccacatgtttgagacgtgccacgcctcacattctccttaccaagtgttgggecceccggttactgtca atataactgagtctgtcaccatatcgtgggegagttcaccgtccttacaacgtgcatatcgaatagcggttttagcctcgcagcagtgcattcttttagcga ccagtgtgcatagtggggtgcggctataattacacaatgacaatttaattaggccegtggatgtctaggctgtatccatttcttctcgacttcgtcagcget ctgggacacacttctttgatcca

XIST, $\mathrm{X}$ inactivate-specific transcript.

1:1,000; ProteinTech Group, Inc.) conjugated with magnetic beads (cat. no. 88802; Pierce; Thermo Fisher Scientific, Inc.) were mixed with the cell lysate and RIP buffer. Isolation of immunoprecipitated RNAs was performed with proteinase K $\left(0.5 \mathrm{mg} / \mathrm{ml}\right.$, EMD Millipore) for $30 \mathrm{~min}$ at $55^{\circ} \mathrm{C}$, and the enrichment of purified RNAs was assessed by RT-qPCR, which was performed as mentioned above.

Luciferase reporter assay. The sequences of XIST or FOXO3 were inserted into the luciferase reporter vector pmirGLO (Promega Corporation) to construct pmirGLO-XIST-wild-type (WT) or pmirGLO-FOXO3-WT reporters. The reporters of pmirGLO-XIST-mutant (MUT) or pmirGLO-FOXO3-MUT were generated by site-directed mutagenesis. Lipofectamine 2000 was used to transfect these plasmids $(1 \mu \mathrm{g})$ with miR-27a-3p mimic or inhibitor and NC mimic and $\mathrm{NC}$ inhibitor $(50 \mathrm{nM})$ into $\mathrm{N} 2 \mathrm{a}$ cells $\left(2 \times 10^{7}\right)$. After transfection for $48 \mathrm{~h}$, the relative luciferase activities were detected using the Luciferase Reporter Assay System (Promega Corporation). The relative luciferase activity was calculated as the ratio of firefly luciferase activity to Renilla luciferase activity.

ELISA. Caspase-3 activity was detected using an ELISA kit (cat. no. MOEB0497; Dakewe Biotech Co., Ltd.) according to the manufacturer's instructions. The optical density values of the reaction product were assessed at $450 \mathrm{~nm}$.
ROS production assay. The transfected N2a cells were stained with $10 \mu \mathrm{M}$ DCFH-DA (Beyotime Institute of Biotechnology) in a $37^{\circ} \mathrm{C}$ bath for $30 \mathrm{~min}$. The ROS activity was analyzed via ImageJ version 6.0 software (National Institutes of Health) and imaged using a Nikon fluorescence microscope (magnification, $\mathrm{x} 100$ ). In total, five wells were randomly selected for each treatment group.

Statistical analysis. Statistical analysis was performed with GraphPad Prism 5 software (GraphPad Software, Inc.). The quantitative data are presented as the mean \pm SD. Statistical significance between two different groups was evaluated using an unpaired Student's t-tests, while differences among $>2$ groups were determined according to one-way ANOVA followed by Tukey's post hoc test. All assays were carried out at $\geq 3$ times with triplicate samples. $\mathrm{P}<0.05$ was considered to indicate a statistically significant difference.

\section{Results}

XIST expression is upregulated in mice with cerebral $I / R$ and in $O G D / R$-treated $N 2 a$ cells. The current study first determined the expression level of XIST in the I/R mouse model and in OGD/R-treated N2a cells using RT-qPCR. The results demonstrated that XIST expression was significantly higher in the cerebral I/R model group compared with that in the sham group (Fig. 1A). Moreover, the expression level 

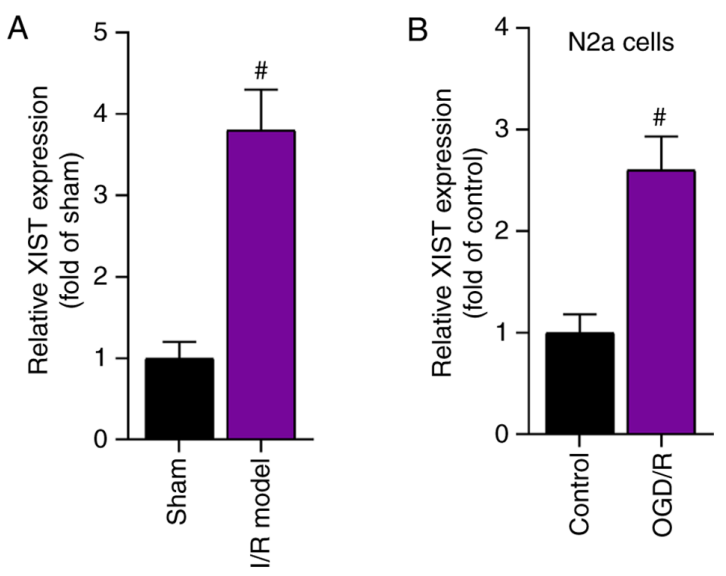

Figure 1. XIST is upregulated in mice with cerebral $I / R$ and in OGD/R-induced N2a cells. (A) RT-qPCR results of the expression level of XIST in mice with cerebral I/R and sham mice. ${ }^{~} \mathrm{P}<0.05$ vs. sham group. (B) RT-qPCR results of the expression levels of XIST in the OGD/R-treated N2a cells and the untreated N2a cells. " $\mathrm{P}<0.05$ vs. control group. RT-qPCR, reverse transcription-quantitative PCR; I/R, ischemia/reperfusion; XIST, $\mathrm{X}$ inactivate-specific transcript; OGD/R, oxygen and glucose deprivation/reperfusion; N2a, Neuro-2a.

of XIST in the OGD/R-treated N2a cells was significantly elevated compared with that in the control group (Fig. 1B). Taken together, the expression level of XIST was upregulated in a cerebral I/R mouse model and in an OGD/R-induced cell model.

Knockdown of XIST inhibits I/R-induced cerebral injury. Whether XIST could influence I/R-induced cerebral injury was investigated. First, the knockdown efficiency of sh-XIST in the brain tissues of the I/R-treated mice was verified (Fig. 2A). Subsequently, TTC staining revealed that the brain infarct volume was significantly increased by I/R treatment; however, the infarct volume was reduced by delivery of AAV-sh-XIST to the brain tissues of the I/R-treated mice (Fig. 2B and C). Moreover, the mice with cerebral I/R developed neurological abnormalities and neurological deficits, and the knockdown of XIST reduced the neurological deficit score in the I/R-treated mice (Fig. 2D).

Cell apoptosis is an important index of cerebral injury; thus, the mRNA and protein expression levels of cell apoptosis-associated genes (Bax and Bcl-2) were detected. On the basis of the RT-qPCR and western blotting data, it was found that the mRNA and protein expression levels of Bax were elevated in the I/R + AVV-sh-NC group compared with the sham + AVV-sh-NC group, and were decreased by XIST knockdown in the mice with cerebral I/R, while the mRNA and protein expression levels of Bcl-2 were reduced in the I/R + AVV-sh-NC group compared with the sham + AVV-sh-NC group, and elevated in the I/R + AVV-sh-XIST group compared with the I/R + AVV-sh-NC group (Fig. 2E and F). In summary, XIST knockdown inhibited cerebral injury induced by $\mathrm{I} / \mathrm{R}$.

XIST interacts with miR-27a-3p. Furthermore, the current study aimed to identify the regulatory mechanisms underlying XIST in cerebral I/R. Given that lncRNAs can function as competitive endogenous RNAs (ceRNAs) by competitively binding with miRNAs to regulate cerebral injury $(22,23)$, we hypothesized that XIST serves as a ceRNA in I/R-induced brain injury. Bioinformatics analysis indicated that XIST can bind with miR-27a-3p, and the putative binding sites for XIST and miR-27a-3p are presented in Fig. 3A.

Subsequently, the overexpression or knockdown efficiency of miR-27a-3p was verified. The RT-qPCR results demonstrated that miR-27a-3p expression was increased by the miR-27a-3p mimic and was decreased by the miR-27a-3p inhibitor (Fig. 3B). Luciferase reporter analysis revealed that overexpression of miR-27a-3p decreased the luciferase activity of the pmirGLO-XIST-WT plasmid, while knockdown of miR-27a-3p increased the luciferase activity of the pmirGLO-XIST-WT plasmid. However, neither miR-27a-3p overexpression or miR-27a-3p knockdown influenced the luciferase activity of the pmirGLO-XIST-Mut plasmid (Fig. 3C), which suggested that XIST bound to miR-27a-3p at the predicted sites. The interaction between XIST and miR-27a-3p was also using by RIP assays, which identified that XIST and miR-27a-3p were abundantly enriched in the Ago2-precipitated products compared with those in the IgG group (Fig. 3D).

Next, RT-qPCR results revealed that XIST was effectively overexpressed or knocked down in N2a cells (Fig. 3E). Overexpression of XIST resulted in a significant reduction in miR-27a-3p expression, while knockdown of XIST caused a significant increase in miR-27a-3p expression (Fig. 3F). Moreover, the expression level of XIST was reduced by miR-27a-3p overexpression and was enhanced by miR-27a-3p knockdown (Fig. 3G). Therefore, the expression level of miR-27a-3p was further assessed in an I/R-induced mouse model and N2a cells via RT-qPCR. The results demonstrated that miR-27a-3p expression was downregulated in the brain tissues of the I/R-treated mice and in the OGD/R-treated N2a cells (Fig. $3 \mathrm{H}$ and I). Overall, these results suggested that XIST interacted with miR-27a-3p.

Overexpression of miR-27a-3p suppresses I/R-induced cerebral injury. As the interaction of XIST and miR-27a-3p was previously confirmed, the function of miR-27a-3p in cerebral $\mathrm{I} / \mathrm{R}$ was further investigated. The overexpression efficiency of miR-27a-3p in the brain tissues of the I/R-treated mice was confirmed via RT-qPCR (Fig. 4A). TTC staining revealed that the brain infarct volume of the I/R-treated mice was reduced by miR-27a-3p overexpression (Fig. 4B and C). Moreover, miR-27a-3p overexpression reduced the neurological deficit score in the I/R-treated mice (Fig. 4D).

To detect the effect of miR-27a-3p on apoptosis, the mRNA and protein expression levels of Bax and Bcl-2 were detected. The RT-qPCR results demonstrated that the Bax mRNA and protein expression levels were decreased, while those of $\mathrm{Bcl}-2$ were elevated by the overexpression of miR-27a-3p in the brain tissues of the I/R-treated mice compared with the I/R + AAV-NC mimic group (Fig. 4E and F). In summary, overexpression of miR-27a-3p suppressed I/R-induced cerebral injury.

XIST regulates the apoptosis and ROS production of N2a cells by binding with miR-27a-3p. Whether XIST regulated the apoptosis and ROS production of N2a cells via interaction with 
B

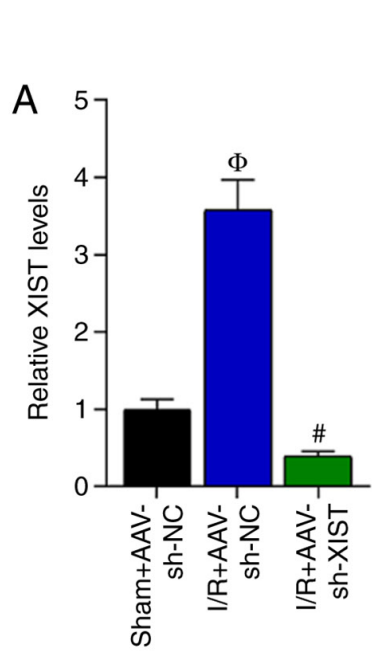

TTC staining

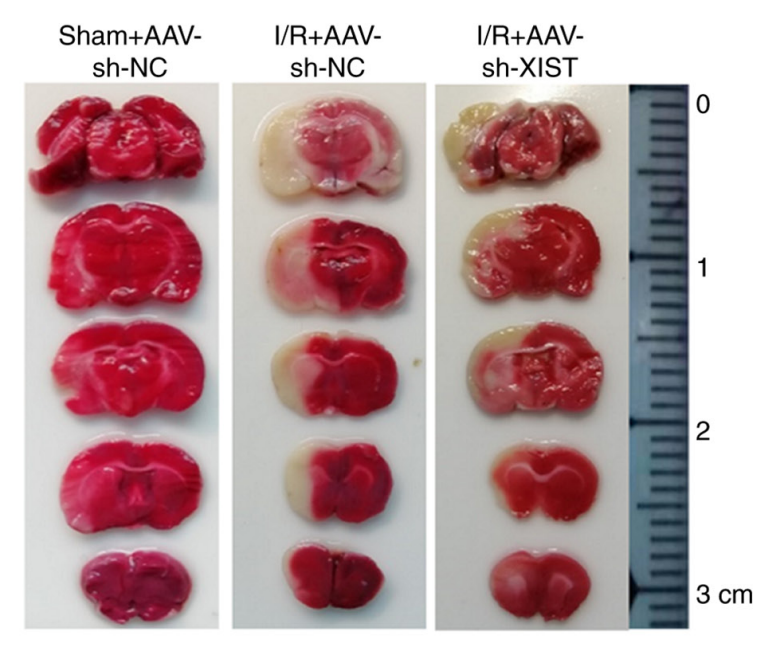

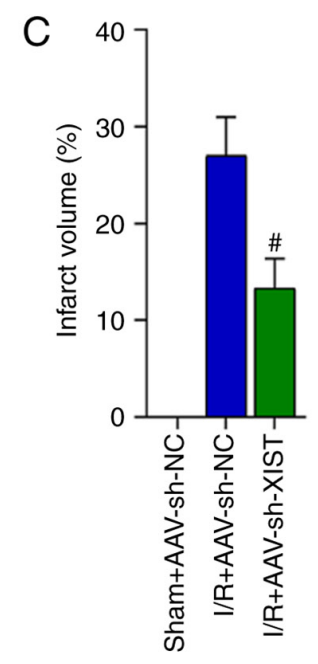
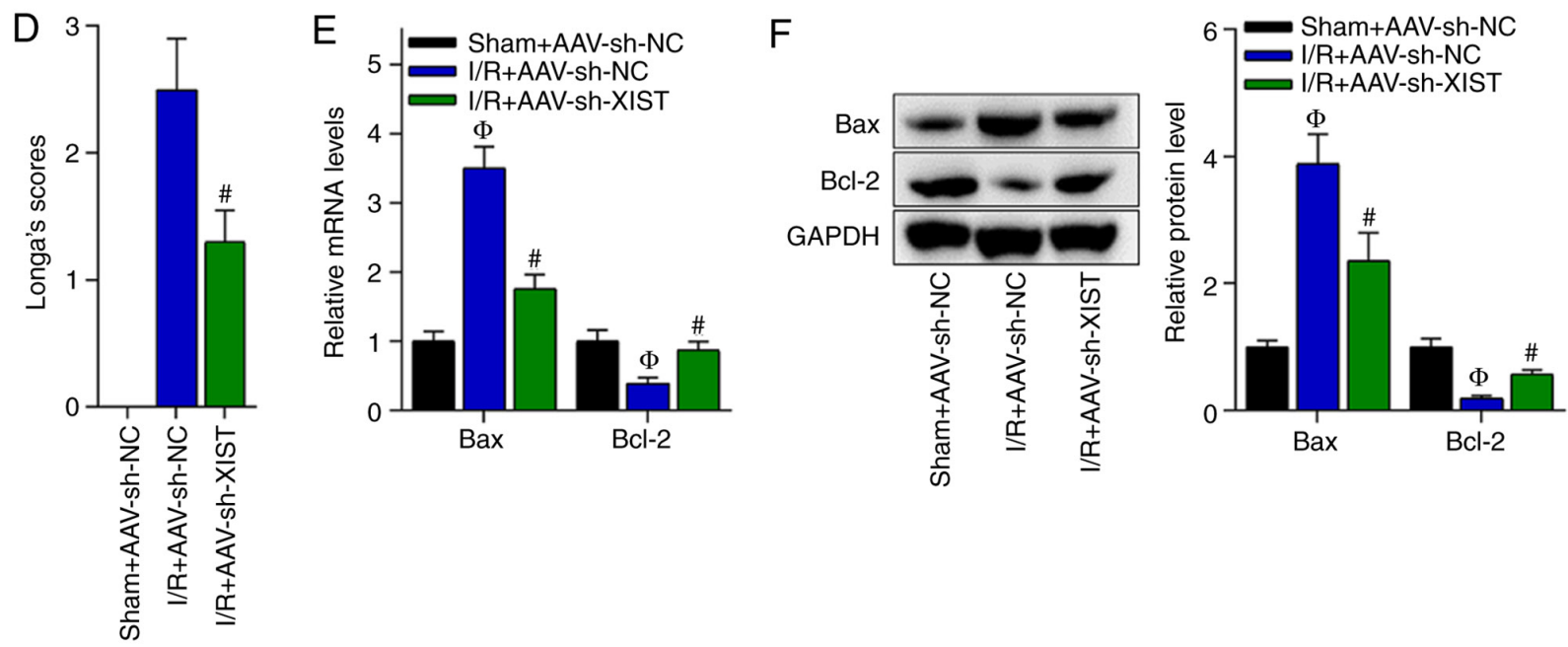

Figure 2. Knockdown of XIST inhibits cerebral injury in I/R-treated mice. (A) Knockdown efficiency of XIST in the brain tissues of I/R-treated mice was assessed via RT-qPCR. ${ }^{\Phi} \mathrm{P}<0.05$ vs. sham $+\mathrm{AAV}$-sh-NC group; ${ }^{~} \mathrm{P}<0.05$ vs. I/R + AAV-sh-NC group. The following three groups were analyzed: Sham + AAV-sh-NC, I/R + AAV-sh-NC and I/R + AAV-sh-XIST. (B) TTC staining images and (C) quantification of the brain infarct volume in the three groups. ${ }^{\#} \mathrm{P}<0.05$ vs. I/R + AAV-sh-NC group. (D) The neurological deficit score was evaluated in the three groups. " $\mathrm{P}<0.05$ vs. I/R + AAV-sh-NC group. (E) RT-qPCR and (F) western blotting were used to detect the mRNA and protein expression levels of Bcl-2 and Bax in the three groups. ${ }^{\Phi} \mathrm{P}<0.05$ vs. sham $+\mathrm{AAV}$-sh-NC group; ${ }^{\text {"P }}<0.05$ vs. I/R + AAV-sh-NC group. NC, negative control; sh, short hairpin RNA; AAV, Adeno-associated virus; RT-qPCR, reverse transcription-quantitative PCR; I/R, ischemia/reperfusion; XIST, X inactivate-specific transcript; TTC, 2,3,5-triphenyltetrazolium chloride.

miR-37a-3p was evaluated. Flow cytometry results indicated that OGD/R induced the apoptosis of N2a cells, while knockdown of XIST decreased cell apoptosis in the OGD/R-treated N2a cells. Furthermore, the repressive effects of XIST knockdown on cell apoptosis were reversed by co-transfection with the miR-37a-3p inhibitor (Fig. 5A and B). It was found that knockdown of XIST decreased the mRNA and protein expression levels of Bax and increased those of Bcl-2 in the OGD/R-treated N2a cells, while knockdown of miR-27a-3p counteracted these effects (Fig. 5C-E). The ELISA results demonstrated that the relative caspase-3 activity and ROS production were enhanced in the supernatant of the OGD/R-treated N2a cells. Moreover, XIST knockdown decreased caspase-3 activity and ROS production compared with the OGD/R group, while knockdown of miR-27a-3p neutralized these inhibitory effects of XIST (Fig. 5F and G). Collectively, it was suggested that XIST promoted the apoptosis and ROS production of N2a cells by binding with miR-27a-3p.
FOXO3 acts as a downstream target of miR-27a-3p. Subsequently, the downstream targets of miR-27a-3p were investigated. Bioinformatics analysis indicated that FOXO3 had binding sites with miR-27a-3p (Fig. 6A). The transfection efficiency of pcDNA3.1-XIST was verified via RT-qPCR in N2a cells (Fig. 6B). Then, pmirGLO-FOXO3-WT luciferase reporter plasmids were constructed by inserting the WT or MUT 3'untranslated region of FOXO3 into pmirGLO plasmids. A luciferase reporter assay revealed that the luciferase activity of pmirGLO-FOXO3-WT was reduced by the overexpression of miR-27a-3p, but was increased by the overexpression of XIST, while the luciferase activity of pmirGLO-FOXO3-MUT showed no evident changes (Fig. 6C). Then, the overexpression efficiency of FOXO3 was confirmed via RT-qPCR in N2a cells transfected with pcDNA3.1-FOXO3 (Fig. 6D). According to RT-qPCR and western blot analyses results, the mRNA and protein expression levels of FOXO3 were downregulated by miR-27a-3p 
A Binding sites of miR-27a-3p on XIST (DIANA)

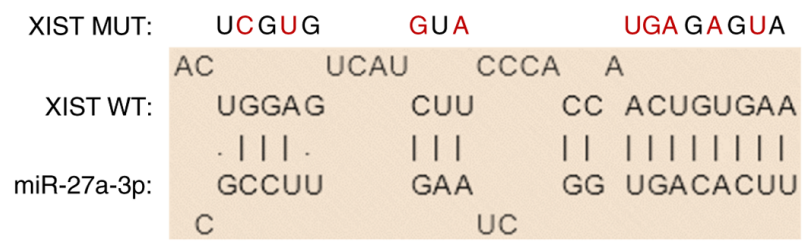

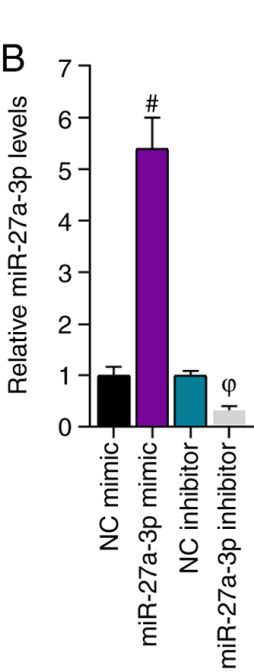
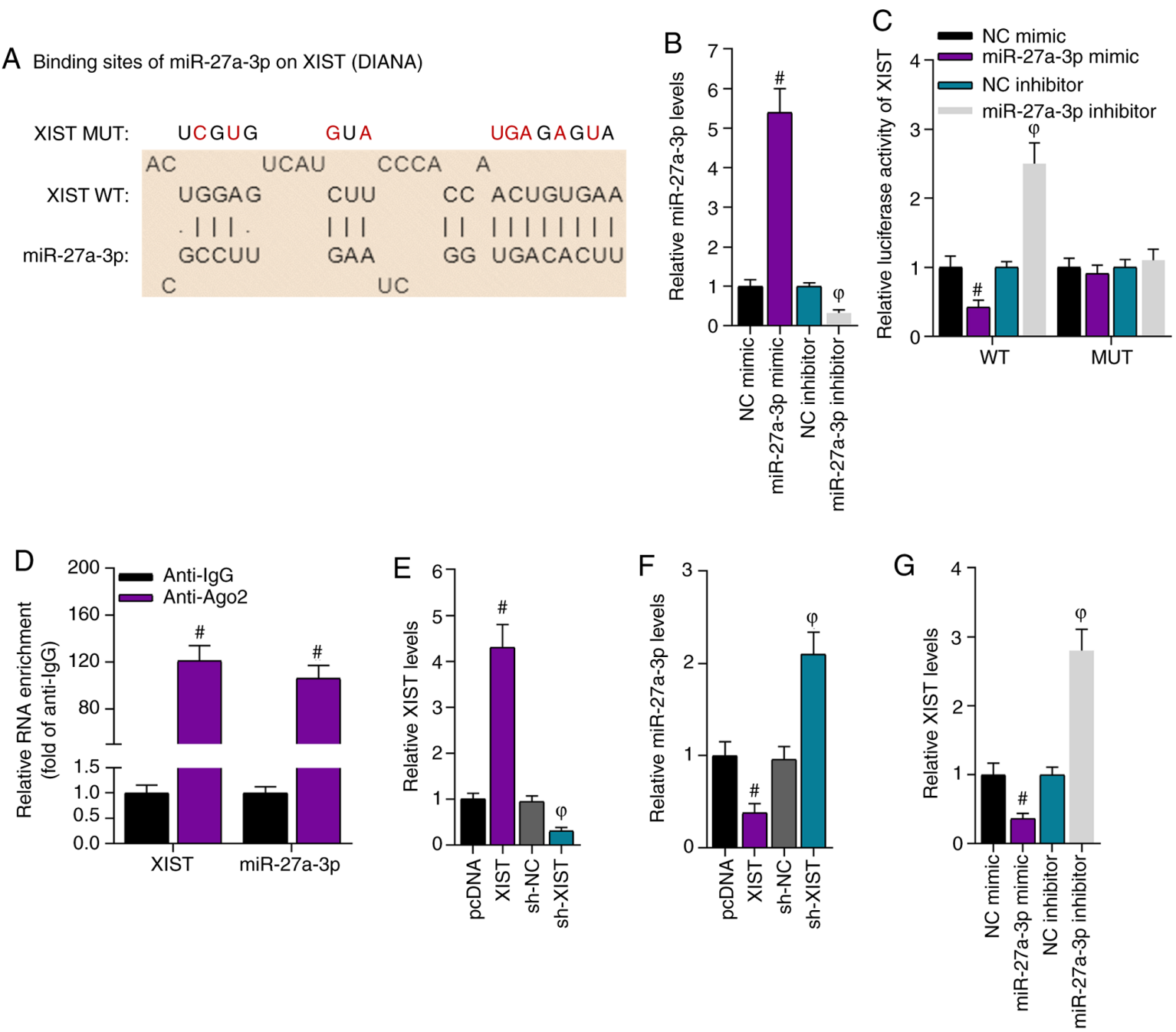
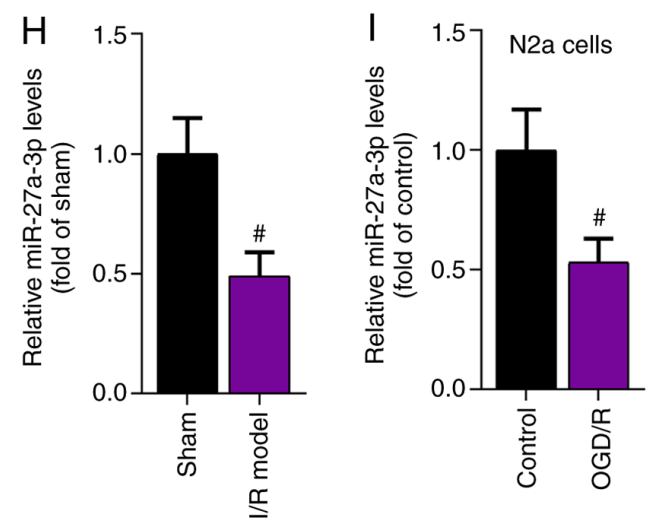

Figure 3. XIST directly interacts with miR-27a-3p. (A) The DIANA database predicted the binding sites between XIST and miR-27a-3p. (B) RT-qPCR results demonstrated the expression level of miR-27a-3p after transfection of miR-27a-3p mimic and miR-27a-3p inhibitor. (C) Luciferase reporter assays revealed the relative luciferase activity of XIST. ${ }^{*} \mathrm{P}<0.05$ vs. NC mimic group; ${ }^{\oplus} \mathrm{P}<0.05$ vs. NC inhibitor group. (D) RNA immunoprecipitation assays showed the relative enrichment of XIST and miR-27a-3p. " $\mathrm{P}<0.05$ vs. anti-IgG group. (E) RT-qPCR determined the overexpression and knockdown efficiency of XIST in N2a cells. (F) RT-qPCR identified the expression level of miR-27a-3p in the N2a cells with overexpressed and silenced XIST. ${ }^{*} \mathrm{P}<0.05$ vs. pcDNA group; ${ }^{\Phi} \mathrm{P}<0.05$ vs. sh-NC group. (G) RT-qPCR results of the expression level of XIST in N2a cells after miR-27a-3p overexpression or knockdown. " $\mathrm{P}<0.05$ vs. NC mimic group; ${ }^{\Phi} \mathrm{P}<0.05$ vs. NC inhibitor group. RT-qPCR results of the expression level of miR-27a-3p in the brain tissues of an (H) I/R injury mouse model and (I) N2a cells. " $\mathrm{P}<0.05$ vs. sham group in panel $\mathrm{H}$; ${ }^{~} \mathrm{P}<0.05$ vs. control group in panel I. RT-qPCR, reverse transcription-quantitative PCR; I/R, ischemia/reperfusion; XIST, X inactivate-specific transcript; NC, negative control; sh, short hairpin RNA; miR, microRNA; WT, wild-type; MUT, mutant; Ago2, argonaute2; OGD/R, oxygen and glucose deprivation/reperfusion; N2a, Neuro-2a.

overexpression and were further enhanced by XIST overexpression (Fig. 6E and F).

Next, the expression level of FOXO3 in the I/R-induced mouse model and the OGD/R-treated N2a cells was assessed.
The results demonstrated that the expression level of FOXO3 was significantly increased in the I/R-induced mouse model and the OGD/R-treated N2a cells (Fig. 6G and H). In summary, it was identified that miR-27a-3p directly bound with FOXO3. 
B

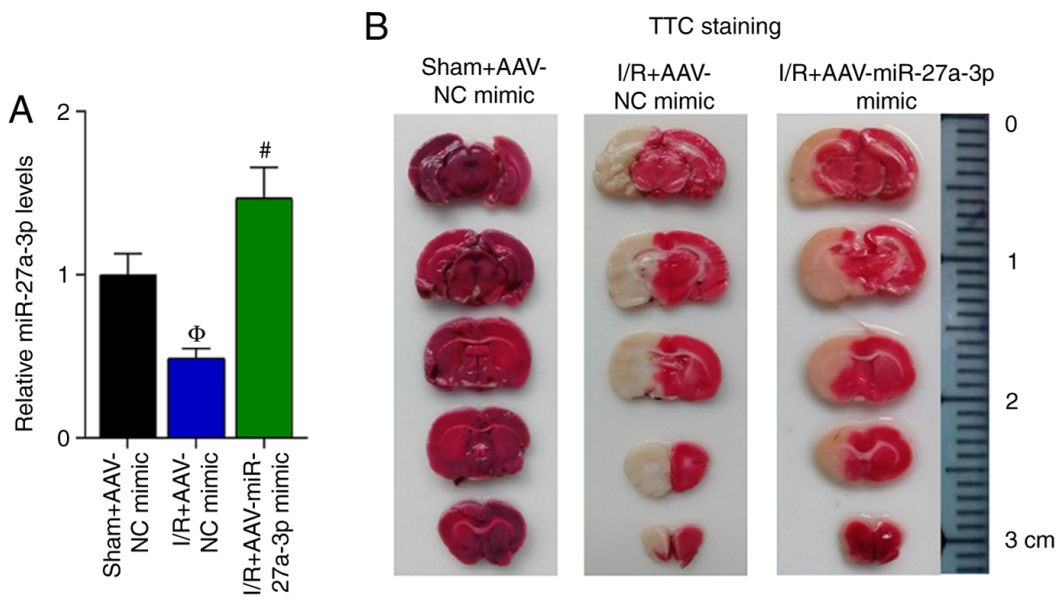

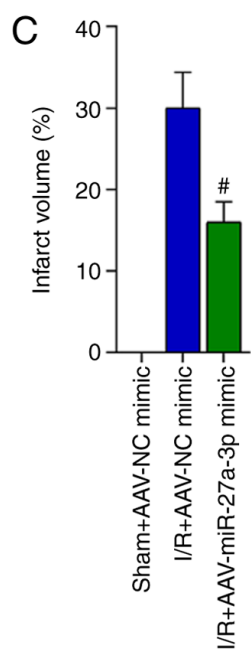

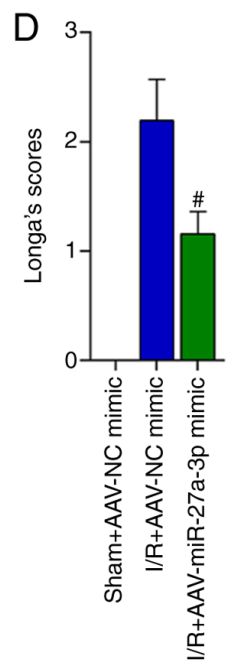

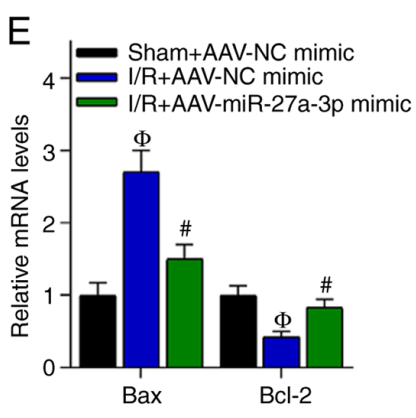

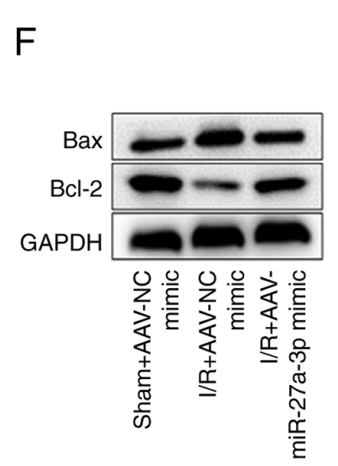

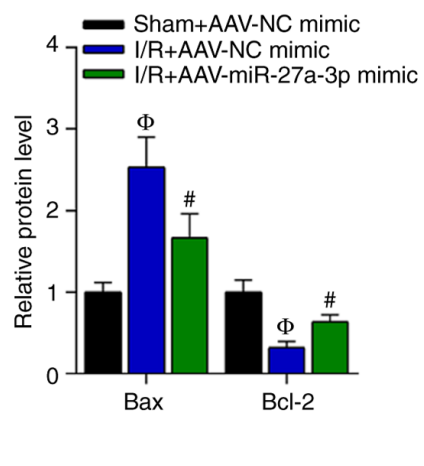

Figure 4. Overexpression of miR-27a-3p suppresses cerebral injury induced by I/R. (A) Overexpression efficiency of miR-27a-3p was detected using RT-qPCR in brain tissues of I/R-treated mice. ${ }^{\Phi} \mathrm{P}<0.05$ vs. sham $+\mathrm{AAV}-\mathrm{NC}$ mimic group; ${ }^{*} \mathrm{P}<0.05$ vs. I/R + AAV-NC mimic group. (B) TTC staining images and (C) quantification of the influence of miR-27a-3p overexpression on brain infarct volume. ${ }^{\#} \mathrm{P}<0.05 \mathrm{vs}$. I/R + AAV-NC mimic group. (D) The neurological deficit score was decreased by miR-27a-3p overexpression. ${ }^{\#} \mathrm{P}<0.05 \mathrm{vs}$. I/R + AAV-NC mimic group. (E) RT-qPCR and (F) western blot analysis revealed the mRNA and protein expression levels of Bax and Bcl-2 after the overexpression of miR-27a-3p. ${ }^{\Phi} \mathrm{P}<0.05 \mathrm{vs}$. sham $+\mathrm{AAV}-\mathrm{NC}$ mimic group; ${ }^{*} \mathrm{P}<0.05 \mathrm{vs}$. I/R $+\mathrm{AAV}-\mathrm{NC}$ mimic group. RT-qPCR, reverse transcription-quantitative PCR; I/R, ischemia/reperfusion; NC, negative control; miR, microRNA; AAV, Adeno-associated virus; TTC, 2,3,5-triphenyltetrazolium chloride.

Knockdown of FOXO3 alleviates cerebral I/R injury. The function of FOXO3 in cerebral injury after I/R was investigated. First, the knockdown efficiency of FOXO3 in the brain tissues of the I/R-treated mice was verified (Fig. 7A). It was identified that FOXO3 knockdown significantly reduced the brain infarct volume in the I/R-treated mice compared with the I/R + AAV-sh-NC group (Fig. 7B and C). Consistently, the neurological deficit score was decreased by the knockdown of FOXO3 in the I/R-treated mice (Fig. 7D). In addition, FOXO3 knockdown decreased the mRNA and protein expression levels of Bax and enhanced those of Bcl-2 in these mice (Fig. 7E and F). In conclusion, the knockdown of FOXO3 alleviated I/R-induced cerebral injury.

\section{Discussion}

Recent studies have revealed that IncRNAs are novel biomarkers in the progression of cerebral I/R injury $(24,25)$.
YY1 transcription factor-induced growth arrest specific 5 promoted 6-phosphofructo-2-kinase/fructose-2,6-biphosphatase 3 transcription to increase neuronal glycolysis, and thus, aggravated cerebral I/R injury (26). Moreover, small nucleolar RNA host gene (SNHG) 12 protected neuronal cells from cerebral I/R injury (27), while SNHG14 accelerated the inflammatory response triggered by cerebral I/R injury by binding with miR-136-5p to regulate Rho associated coiled-coil containing protein kinase (28).

XIST has been reported to serve a role in human lung adenocarcinoma (13), pulmonary fibrosis (14) and neuropathic pain (15), but the exact function of XIST in cerebral I/R injury remains unknown. The present study identified that XIST expression was upregulated in the brain tissues of an I/R mouse model and in OGD/R-induced N2a cells. Furthermore, it was found that XIST knockdown inhibited brain injury by suppressing apoptosis and ROS production. 

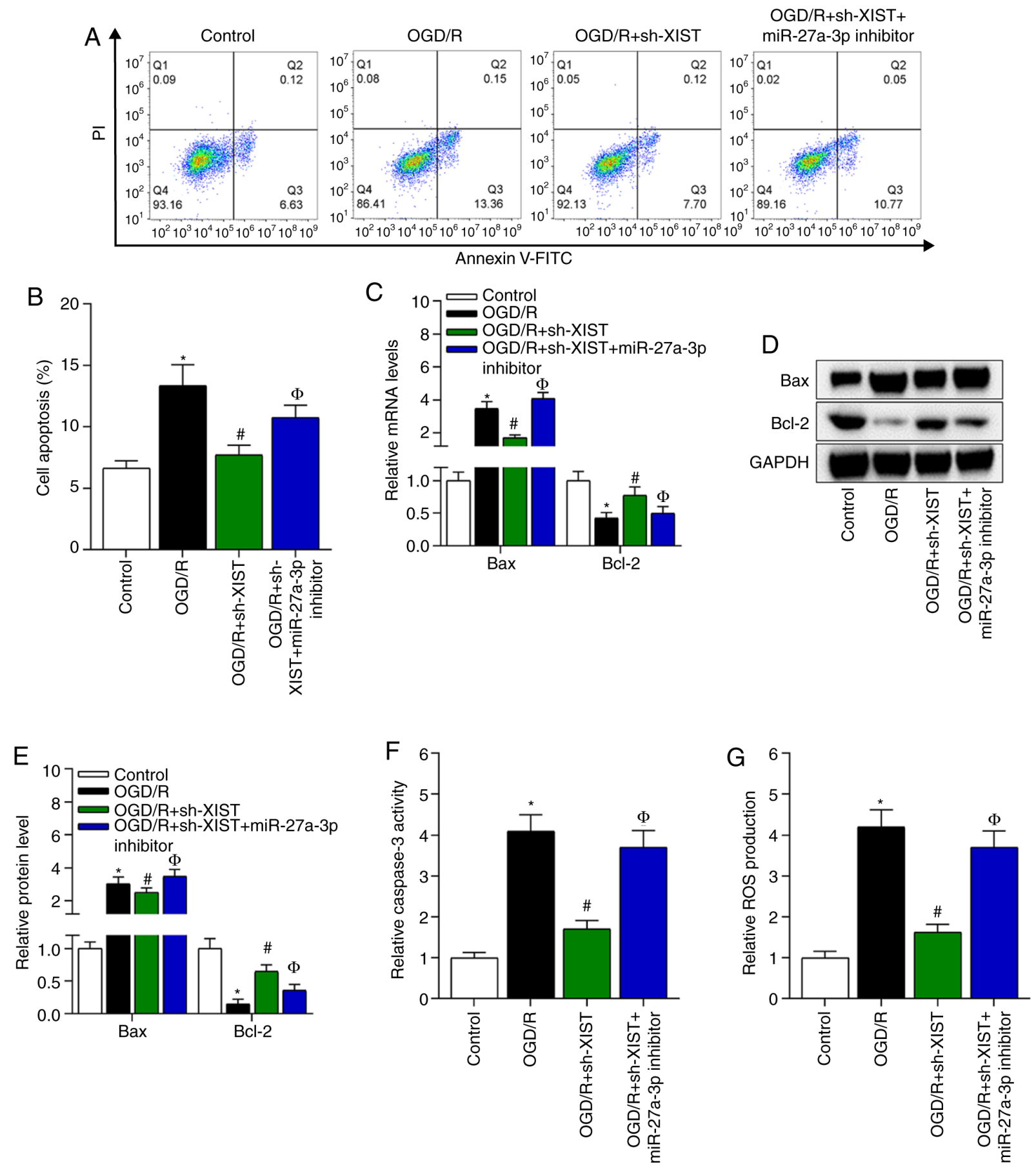

ROS

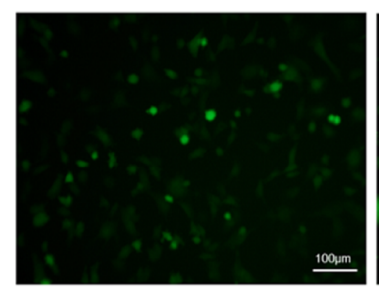

Control

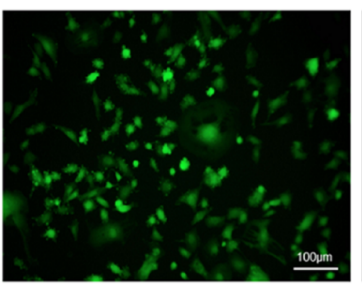

OGD/R

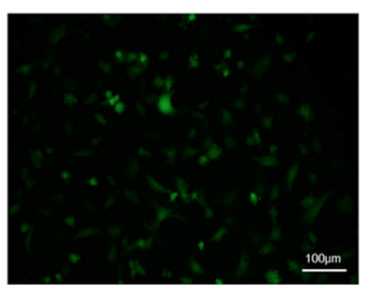

OGD/R+sh-XIST

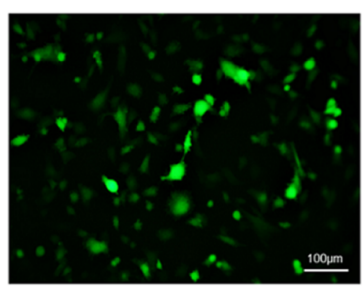

OGD/R+sh-XIST+ miR-27a-3p inhibitor

Figure 5. XIST regulates the apoptosis of N2a cells by binding with miR-27a-3p. (A) Flow cytometry was used to measure N2a cell apoptosis, and (B) the percentage of cell apoptosis was quantified. " $\mathrm{P}<0.05$ vs. control group; ${ }^{\prime \prime} \mathrm{P}<0.05$ vs. OGD/R group; ${ }^{\oplus} \mathrm{P}<0.05$ vs. OGD/R + sh-XIST group. (C) Reverse transcription-quantitative PCR and (D) western blot analysis of the mRNA and (E) protein expression levels of Bax and Bcl-2 in N2a cells. ELISA results of the relative (F) caspase-3 activity. (G) ROS production changes in the OGD/R-treated N2a cells. The ROS staining images in the four indicated groups for panel G. Scale bar, $100 \mu \mathrm{m}$. " $\mathrm{P}<0.05$ vs. control group; ${ }^{\prime} \mathrm{P}<0.05$ vs. OGD/R group; ${ }^{\Phi} \mathrm{P}<0.05$ vs. OGD/R + sh-XIST group. I/R, ischemia/reperfusion; NC, negative control; miR, microRNA; OGD/R, oxygen and glucose deprivation/reperfusion; N2a, Neuro-2a; sh, short hairpin RNA; XIST, X inactivate-specific transcript; ROS, reactive oxygen species. 


\begin{tabular}{|c|c|}
\hline FOXO3 3' UTR MUT & $5^{\prime}$...CAGUGUACCCCAUUAAGUCUCAU.... $3^{\prime}$ \\
\hline & $\begin{array}{l}\text { Predicted consequential pairing of target region (top) } \\
\text { and miRNA (bottom) }\end{array}$ \\
\hline $\begin{array}{l}\text { Position } 3257-3264 \text { of FOXO3 3' UT } \\
\text { hsa-miR-27a-3p }\end{array}$ & 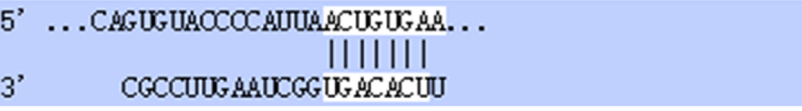 \\
\hline
\end{tabular}

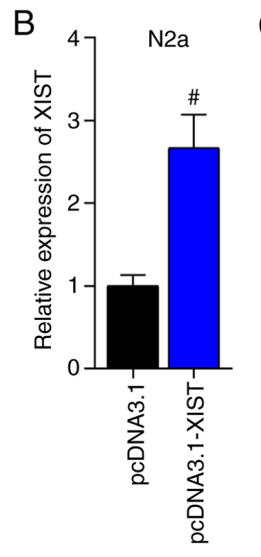

$\mathrm{F}$

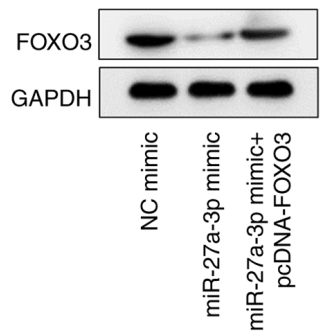

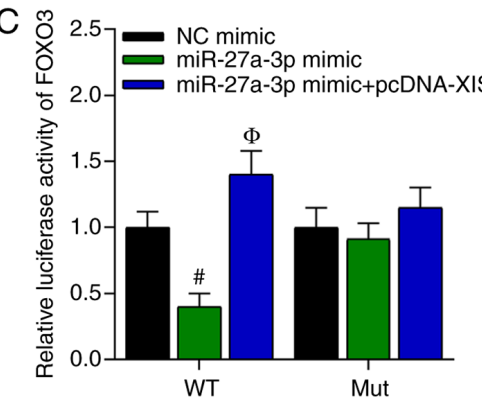

WT

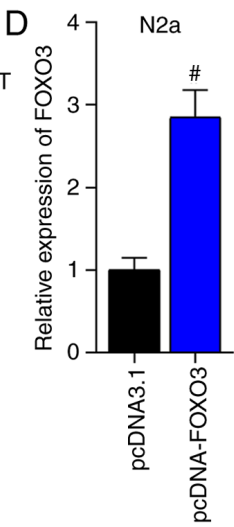

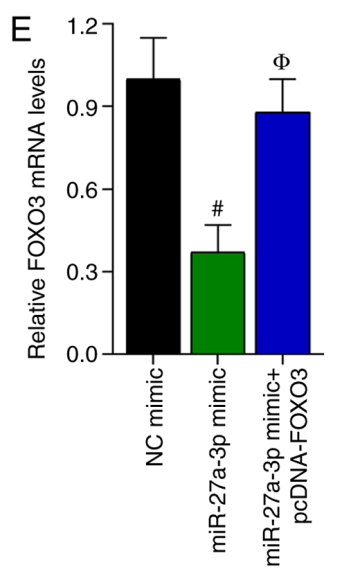
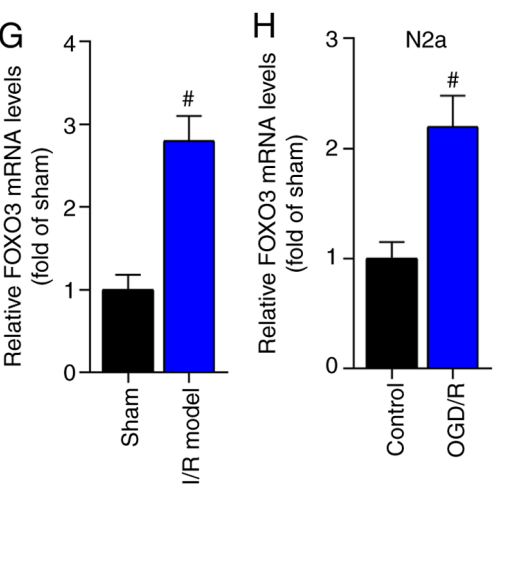

Figure 6. FOXO3 acts as a downstream target of miR-27a-3p. (A) Bioinformatics analysis indicated that FOXO3 had binding sites for miR-27a-3p. (B) The overexpression efficiency of XIST was detected using RT-qPCR in N2a cells. ${ }^{*} \mathrm{P}<0.05$ vs. pcDNA3.1 group. (C) A luciferase reporter assay revealed the luciferase activity of FOXO3. ${ }^{*} \mathrm{P}<0.05$ vs. $\mathrm{NC}$ mimic group; ${ }^{\oplus \mathrm{P}}<0.05$ vs. miR-27a-3p mimic group. (D) Transfection efficiency of FOXO3 was examined via RT-qPCR in N2a cells transfected with pcDNA3.1-FOXO3. ${ }^{\sharp} \mathrm{P}<0.05$ vs. pcDNA3.1 group. (E) RT-qPCR and (F) western blot analysis results demonstrated the mRNA and protein expression level changes of FOXO3 in N2a cells. ${ }^{~} \mathrm{P}<0.05$ vs. NC mimic group; ${ }^{\oplus} \mathrm{P}<0.05$ vs. miR-27a-3p mimic group. RT-qPCR was used to measure the expression of FOXO3 in the $(\mathrm{G})$ brain tissues of the I/R-treated mice and the $(\mathrm{H}) \mathrm{OGD} / \mathrm{R}$-treated $\mathrm{N} 2 \mathrm{a}$ cells. ${ }^{~} \mathrm{P}<0.05$ vs. sham group in panel G; ${ }^{*} \mathrm{P}<0.05$ vs. control group in panel H. N2a, Neuro-2a; XIST, X inactivate-specific transcript; I/R, ischemia/reperfusion; NC, negative control; miRNA/miR, microRNA; OGD/R, oxygen and glucose deprivation/reperfusion; RT-qPCR, reverse transcription-quantitative PCR; WT, wild-type; MUT, mutant; UTR, untranslated region.

IncRNAs can serve as ceRNAs to regulate mRNA expression by competitively binding with shared miRNAs in brain injury. For example, AK038897 functions as a ceRNA against miR-26a-5p to upregulate death associated protein kinase 1 and aggravate cerebral I/R injury (29). It has also been shown that myocardial infarction associated transcript competed with high mobility group box 1 to bind with miR-204-5p, and thereby, regulated cerebral microvascular endothelial cell injury after cerebral ischemia (30). Furthermore, SNHG6 modulated neuronal cell apoptosis by modulating miR-181c-5p/Bcl-2-like protein 11 signaling in ischemic stroke (31). Using bioinformatics analysis and mechanistic assays, miR-27a-3p was revealed to bind with XIST in the present study. It has been verified that miR-27a-3p suppresses the progression of occlusive bronchiolitis (31), and upregulation of miR-27a-3p inhibits the inflammatory response in spinal cord injury by targeting Toll-like receptor 4 (32). The present study demonstrated that miR-27a-3p was downregulated in the brain tissues of an I/R mouse model and in OGD/R-treated N2a cells. In addition, overexpression of miR-27a-3p attenuated brain injury induced by $\mathrm{I} / \mathrm{R}$ in mice. It was found that apoptosis and ROS production in N2a cells were suppressed by the overexpression of miR-27a-3p. Moreover, the rescue assays demonstrated that XIST facilitated cerebral I/R injury by downregulating miR-27a-3p.

In the current study, $\mathrm{FOXO} 3$ was confirmed to be a downstream target of miR-27a-3p. FOXO3 was previously reported to regulate pulmonary fibrosis (33) and inflammation in mice with necrotizing colitis (34). The present results indicated that FOXO3 expression was negatively regulated by $\mathrm{miR}-27 \mathrm{a}-3 \mathrm{p}$ and was positively regulated by XIST. Furthermore, FOXO3 expression was significantly upregulated in the brain tissues of the I/R-treated mice and in the OGD/R-induced N2a cells. More importantly, 

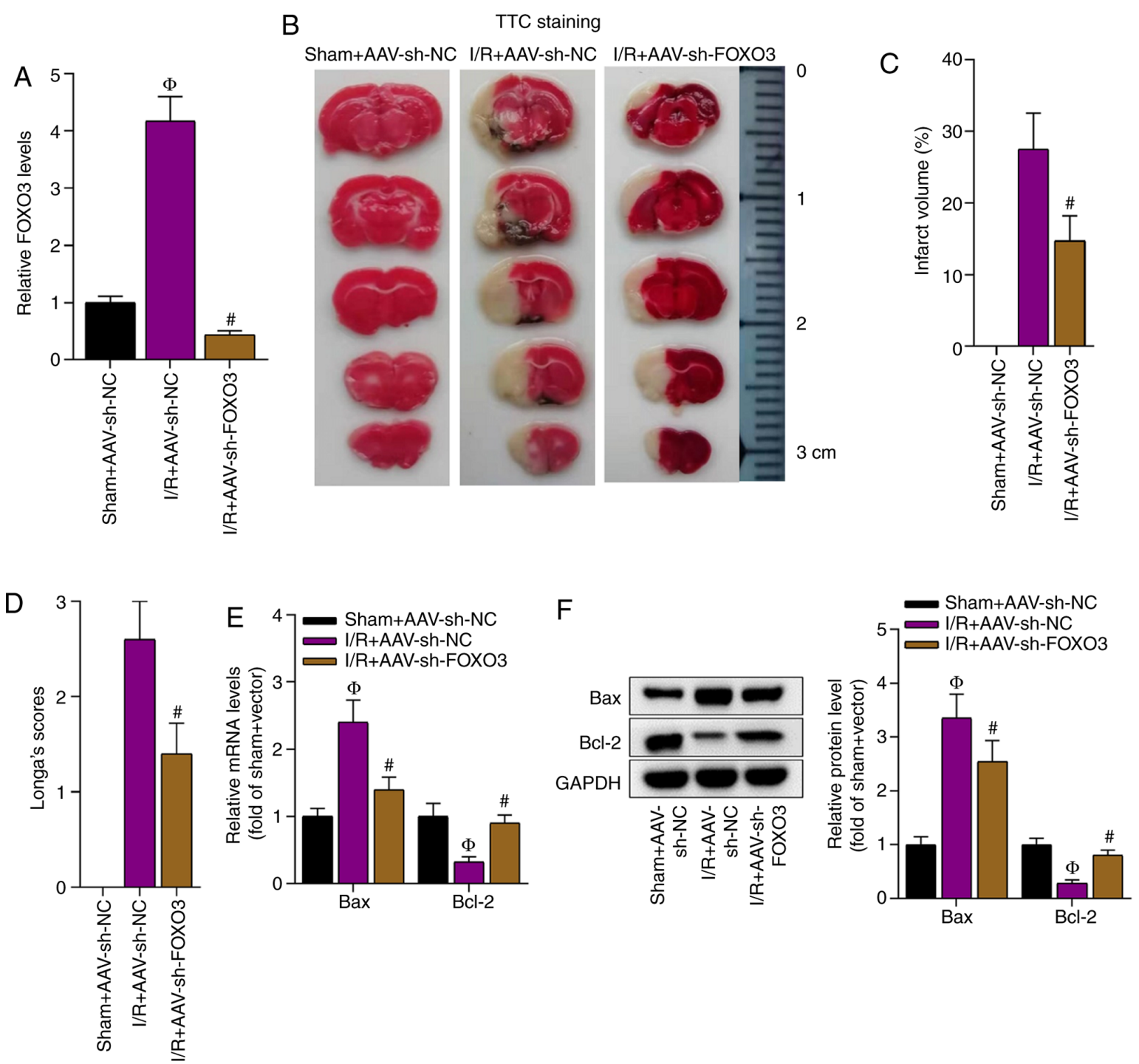

Figure 7. Knockdown of FOXO3 alleviates cerebral I/R injury. (A) Knockdown efficiency of FOXO3 in brain tissues of I/R-treated mice was detected via RT-qPCR. ${ }^{\Phi} \mathrm{P}<0.05$ vs. sham $+\mathrm{AAV}$-sh-NC group; ${ }^{*} \mathrm{P}<0.05$ vs. I/R + AAV-sh-NC group. (B) TTC staining images and (C) quantification of the brain infarct volume after knockdown of FOXO3. ${ }^{~} \mathrm{P}<0.05$ vs. I/R + AAV-sh-NC group. (D) The influence of FOXO3 knockdown on the neurological deficit score. ${ }^{\#} \mathrm{P}<0.05$ vs. I/R + AAV-sh-NC group. (E) RT-qPCR and (F) western blot analysis results of the mRNA and protein expression levels of Bax and Bcl-2 after the knockdown of FOXO3. ${ }^{\Phi} \mathrm{P}<0.05$ vs. sham + AAV-sh-NC group; ${ }^{~} \mathrm{P}<0.05$ vs. I/R + AAV-sh-NC group. I/R, ischemia/reperfusion; NC, negative control; RT-qPCR, reverse transcription-quantitative PCR; sh, short hairpin RNA; AAV, Adeno-associated virus; TTC, 2,3,5-triphenyltetrazolium chloride.

FOXO3 knockdown alleviated I/R-induced injury, as well as suppressed the apoptosis and ROS production of N2a cells.

In conclusion, the present study demonstrated that XIST promoted cerebral I/R injury by binding with miR-27a-3p to upregulate $\mathrm{FOXO} 3$, which may further the understanding of the pathogenesis of cerebral I/R injury.

\section{Acknowledgements}

Not applicable.

\section{Funding}

This study was supported by Key Project Fund for Clinical Research of Wuhan Health and Family Planning Commission (grant no. WX15B22), Young Talents Project Fund of Hubei Municipal Health Commission (grant no. WJ2019H168) and Wuhan Young and Middle-Aged Medical Backbone Talents
Training Project Fund [Wuhan Health and Family Planning Commission (2017); no. 51].

\section{Availability of data and materials}

The datasets used and/or analyzed during the current study are available from the corresponding author on reasonable request.

\section{Authors' contributions}

$\mathrm{HZ}$ and $\mathrm{XW}$ conceived and designed the experiments. HZ, JX, QH, LX, HC and MC performed the experiments and constructed the table and figures. HZ, JX, QH and XW provided the reagents, materials and analysis tools. $\mathrm{HZ}$ and XW wrote the paper. HZ, JX, QH, LX and XW revised the manuscript. All authors read and approved the final version of the manuscript. All authors confirm the authenticity of all the raw data. 


\section{Ethics approval and consent to participate}

The study protocols of all animal experiments were approved and performed in accordance with standard principles approved by the Institutional Animal Care and Use Committee of China Resources \& WISCO General Hospital (Hubei, China).

\section{Patient consent for publication}

Not applicable.

\section{Competing interests}

The authors declare that they have no competing interests.

\section{References}

1. Ou J, Kou L, Liang L and Tang C: MiR-375 attenuates injury of cerebral ischemia/reperfusion via targetting Ctgf. Biosci Rep 37: BSR20171242, 2017.

2. Henriksson KM, Farahmand B, Åsberg S, Edvardsson N and Terént A: Comparison of cardiovascular risk factors and survival in patients with ischemic or hemorrhagic stroke. Int J Stroke 7: 276-281, 2012.

3. Ma J, Shui S, Han X, Guo D, Li T and Yan L: microRNA-200a silencing protects neural stem cells against cerebral ischemia/reperfusion injury. PLoS One 12: e0172178, 2017.

4. Dorweiler B, Pruefer D, Andrasi TB, Maksan SM, Schmiedt W and Neufang A and Vahl CF: Ischemia-reperfusion injury: Pathophysiology and clinical implications. Eur J Trauma Emerg Surg 33: 600-612, 2007.

5. Pan J, Konstas AA, Bateman B, Ortolano GA and Pile-Spellman J: Reperfusion injury following cerebral ischemia: Pathophysiology, MR imaging, and potential therapies. Neuroradiology 49: 93-102, 2007.

6. Schrepfer E and Scorrano L: Mitofusins, from mitochondria to metabolism. Mol Cell 61: 683-694, 2016.

7. Khoshnam SE, Winlow W, Farzaneh M, Farbood Y and Moghaddam HF: Pathogenic mechanisms following ischemic stroke. Neurol Sci 38: 1167-1186, 2017.

8. Ma L, Bajic VB and Zhang Z: On the classification of long non-coding RNAs. RNA Biol 10: 925-933, 2013

9. Kapranov P,Cheng J,DikeS,Nix DA,Duttagupta R,WillinghamAT, Stadler PF, Hertel J, Hackermüller J, Hofacker IL, et al: RNA maps reveal new RNA classes and a possible function for pervasive transcription. Science 316: 1484-1488, 2007.

10. Ge Y, Wang J, Wu D, Zhou Y, Qiu S, Chen J, Zhu X, Xiang X, Li H and Zhang D: IncRNA NR_038323 Suppresses renal fibrosis in diabetic nephropathy by targeting the miR-324-3p/DUSP1 axis Mol Ther Nucleic Acids 17: 741-753, 2019.

11. Zhang G, Li S, Lu J, Ge Y, Wang Q, Ma G, Zhao Q, Wu D, Gong W, Du M, et al: LncRNA MT1JP functions as a ceRNA in regulating FBXW7 through competitively binding to miR-92a-3p in gastric cancer. Mol Cancer 17: 87, 2018.

12. Li D, Zhang J, Li X, Chen Y, Yu F and Liu Q: Insights into lncRNAs in Alzheimer's disease mechanisms. RNA Biol: Jul 14, 2020 (Epub ahead of print). doi: 10.1080/15476286.2020.1788848

13. Sun J, Pan LM, Chen LB and Wang Y: LncRNA XIST promotes human lung adenocarcinoma cells to cisplatin resistance via let-7i/BAG-1 axis. Cell Cycle 16: 2100-2107, 2017.

14. Wang Y, Liang Y, Luo J, Nie J, Yin H, Chen Q, Dong J, Zhu J, Xia J and Shuai W: XIST/miR-139 axis regulates bleomycin (BLM)-induced extracellular matrix (ECM) and pulmonary fibrosis through $\beta$-catenin. Oncotarget 8: 65359-65369, 2017.

15. Jin H, Du XJ, Zhao Y and Xia DL: XIST/miR-544 axis induces neuropathic pain by activating STAT3 in a rat model. J Cell Physiol 233: 5847-5855, 2018.

16. Longa EZ, Weinstein PR, Carlson S and Cummins R: Reversible middle cerebral artery occlusion without craniectomy in rats. Stroke 20: 84-91, 1989.
17. Ansari S, Azari H, Caldwell KJ, Regenhardt RW, Hedna VS, Waters MF, Hoh BL and Mecca AP: Endothelin-1 induced middle cerebral artery occlusion model for ischemic stroke with laser Doppler flowmetry guidance in rat. J Vis Exp: 50014, 2013 doi: $10.3791 / 50014$.

18. Hu X, Xiang Z, Zhang W, Yu Z, Xin X, Zhang R, Deng Y and Yuan Q: Protective effect of DLX6-AS1 silencing against cerebral ischemia/reperfusion induced impairments. Aging (Albany NY) 12: 23096-23113, 2020.

19. Lu Y,Han Y,He J,Zhou B, Fang P and Li X: LncRNA FOXD3-AS1 knockdown protects against cerebral ischemia/reperfusion injury via miR-765/BCL2L13 axis. Biomed Pharmacother 132: 110778, 2020.

20. Cao Y, Gao W, Tang H, Wang T and You C: Long Non-coding RNA TALNEC2 Aggravates Cerebral Ischemia/Reperfusion Injury via Acting as a competing endogenous RNAs for miR-650 to target apoptotic peptidase activating factor 1 . Neuroscience 458: 64-76, 2021.

21. Livak KJ and Schmittgen TD: Analysis of relative gene expression data using real-time quantitative PCR and the 2(-Delta Delta C(T)) method. Methods 25: 402-408, 2001.

22. Gao N, Tang H, Gao L, Tu GL, Luo H and Xia Y: LncRNA H19 aggravates cerebral ischemia/reperfusion injury by functioning as a ceRNA for miR-19a-3p to Target PTEN. Neuroscience 437: $117-129,2020$.

23. Shan W, Chen W, Zhao X, Pei A, Chen M, Yu Y, Zheng Y and Zhu S: Long noncoding RNA TUG1 contributes to cerebral ischaemia/reperfusion injury by sponging mir-145 to up-regulate AQP4 expression. J Cell Mol Med 24: 250-259, 2020.

24. Ghafouri-Fard S, Shoorei H and Taheri M: Non-coding RNAs participate in the ischemia-reperfusion injury. Biomed Pharmacother 129: 110419, 2020.

25. Liang J, Wang Q, Li JQ, Guo T and Yu D: Long non-coding RNA MEG3 promotes cerebral ischemia-reperfusion injury through increasing pyroptosis by targeting miR-485/AIM2 axis. Exp Neurol 325: 113139, 2020.

26. Zhang XC, Gu AP, Zheng CY, Li YB, Liang HF, Wang HJ, Tang XL, Bai XX and Cai J: YY1/LncRNA GAS5 complex aggravates cerebral ischemia/reperfusion injury through enhancing neuronal glycolysis. Neuropharmacology 158: 107682, 2019.

27. Yao X, Yao R, Huang F and Yi J: LncRNA SNHG12 as a potent autophagy inducer exerts neuroprotective effects against cerebral ischemia/reperfusion injury. Biochem Biophys Res Commun 514: 490-496, 2019.

28. Zhong Y, Yu C and Qin W: LncRNA SNHG14 promotes inflammatory response induced by cerebral ischemia/reperfusion injury through regulating miR-136-5p /ROCK1. Cancer Gene Ther 26: 234-247, 2019.

29. Wei R, Zhang L, Hu W, Wu J and Zhang W: Long non-coding RNA AK038897 aggravates cerebral ischemia/reperfusion injury via acting as a ceRNA for miR-26a-5p to target DAPK1. Exp Neurol 314: 100-110, 2019.

30. Deng W, Fan C, Shen R, Wu Y, Du R and Teng J: Long noncoding MIAT acting as a ceRNA to sponge microRNA-204-5p to participate in cerebral microvascular endothelial cell injury after cerebral ischemia through regulating HMGB1. J Cell Physiol 235: 4571-4586, 2020.

31. Zhang X, Liu Z, Shu Q, Yuan S, Xing Z and Song J: LncRNA SNHG6 functions as a ceRNA to regulate neuronal cell apoptosis by modulating miR-181c-5p/BIM signalling in ischaemic stroke. J Cell Mol Med 23: 6120-6130, 2019.

32. Zhang P, Li LQ, Zhang D and Shen Y: Over-expressed miR-27a-3p inhibits inflammatory response to spinal cord injury by decreasing TLR4. Eur Rev Med Pharmacol Sci 22: 5416-5423, 2018.

33. Qian W, Cai X, Qian Q, Wang D and Zhang L: Angelica sinensis polysaccharide suppresses epithelial-mesenchymal transition and pulmonary fibrosis via a DANCR/AUF-1/FOXO3 regulatory axis. Aging Dis 11: 17-30, 2020.

34. Yin Y, Wang J, Zhao X, Wu X, Zou H, Qin Z and Cao J: Overexpressed FOXO3 improves inflammatory status in mice by affecting NLRP3-mediated cell coronation in necrotizing colitis mice. Biomed Pharmacother 125: 109867, 2020.

This work is licensed under a Creative Commons Attribution-NonCommercial-NoDerivatives 4.0 International (CC BY-NC-ND 4.0) License. 